\title{
Possible splitting of deconfinement and chiral transitions in strong magnetic fields in QCD
}

\author{
Eduardo S. Fraga* \\ Instituto de Física, Universidade Federal do Rio de Janeiro, Caixa Postal 68528, Rio de Janeiro, \\ RJ 21941-972, Brazil \\ E-mail: fragaeif.ufrj.br

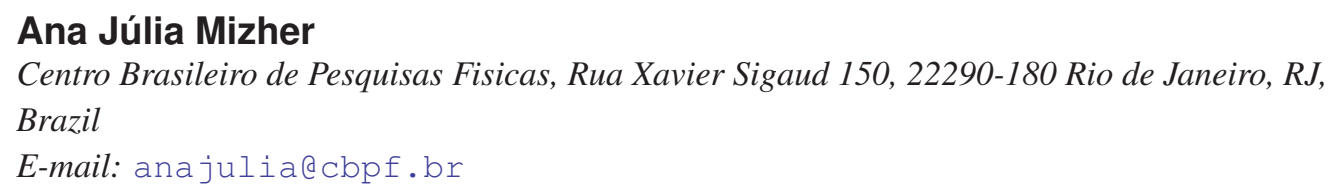

\section{N. Chernodub ${ }^{\dagger}$}

CNRS, Laboratoire de Mathématiques et Physique Théorique, Université François-Rabelais, Fédération Denis Poisson - CNRS, Parc de Grandmont, Université de Tours, 37200 France Department of Physics and Astronomy, University of Gent, Krijgslaan 281, S9, B-9000 Gent, Belgium

E-mail: maxim. chernodubelmpt.univ-tours.fr

We show that finite-temperature deconfinement and chiral transitions can split in a strong enough magnetic field. The splitting in critical temperatures of these transitions in a constant magnetic field of a typical LHC magnitude is of the order of $10 \mathrm{MeV}$. A new deconfined phase with broken chiral symmetry appears.

35th International Conference of High Energy Physics - ICHEP2010,

July 22-28, 2010

Paris France

\footnotetext{
*Speaker.

$\dagger$ On leave from ITEP, Moscow, Russia.
} 
The thermal quark-hadron transition can be dramatically modified in the presence of a strong magnetic field $[1,2,3,4,5,6]$. We work in the two-flavor linear sigma model coupled to quarks and to the Polyakov loop $\left(\mathrm{PLSM}_{q}\right)$ in the presence of an external magnetic field [5]. The confining properties of QCD are described by the complex-valued Polyakov loop variable $L$. The expectation value of the Polyakov loop $L$ is an exact order parameter of the color confinement in the limit of infinitely massive quarks:

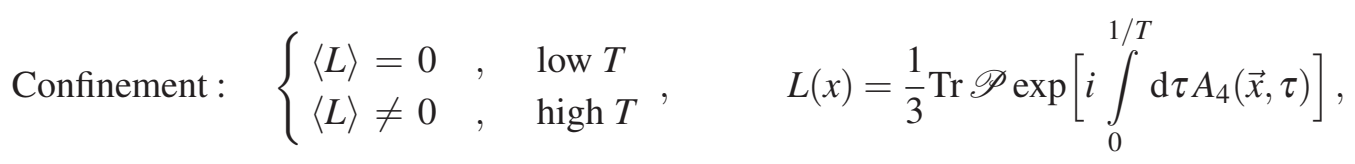

where $A_{4}=i A_{0}$ is the matrix-valued temporal component of the Euclidean gauge field $A_{\mu}$ and the symbol $\mathscr{P}$ denotes path ordering. The integration takes place over compactified imaginary time $\tau$.

The chiral features of the model are encoded in the dynamics of the $O(4)$ chiral field, the singlet component of which is an exact order parameter in the chiral limit (i.e., when quarks and pions are massless degrees of freedom):

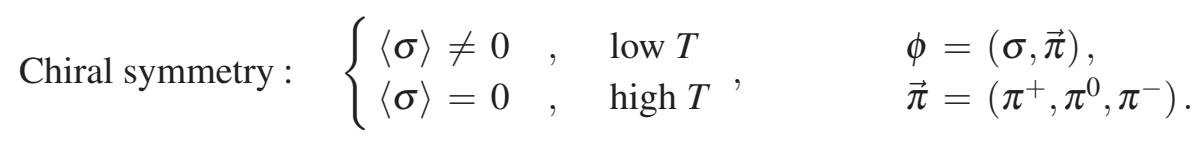

Here $\vec{\pi}$ is the isotriplet of the pseudoscalar pion fields and $\sigma$ is the chiral scalar field which plays the role of an approximate order parameter of the chiral transition in QCD.

The quark field $\psi$ provides interaction between the Polyakov loop $L$ and the chiral field $\phi$, making a bridge between confining and chiral properties. Quarks are also coupled to the external magnetic field since the $u$ and $d$ quarks are electrically charged. Thus, it is clear that the external magnetic field will affect the chiral dynamics and the confining properties of the model.

The Lagrangian of $\operatorname{PLSM}_{q}$ describes the constituent quarks $\psi$, which interact with the meson fields $\sigma, \pi^{ \pm}=\left(\pi^{1} \pm i \pi^{2}\right) / \sqrt{2}$ and $\pi^{0}=\pi^{3}$, the Abelian gauge field $a_{\mu}=\left(a^{0}, \vec{a}\right)=(0,-B y, 0,0)$, and the $S U(3)$ gauge field $A_{\mu}$ (related to the Polyakov loop $L$, see Eq. (1)) via the covariant derivative $\boldsymbol{D}=\gamma^{\mu}\left(\partial_{\mu}-i Q a_{\mu}-i A_{\mu}\right)$ with the charge matrix $Q=\operatorname{diag}(+2 e / 3,-e / 3)$ :

$$
\mathscr{L}=\bar{\psi}\left[i D-g\left(\sigma+i \gamma_{5} \vec{\tau} \cdot \vec{\pi}\right)\right] \psi+\frac{1}{2}\left[\left(\partial_{\mu} \sigma\right)^{2}+\left(\partial_{\mu} \pi^{0}\right)^{2}\right]+\left|D_{\mu}^{(\pi)}\right|^{2}-V_{\phi}(\sigma, \vec{\pi})-V_{L}(L, T),
$$

where $D_{\mu}^{(\pi)}=\partial_{\mu}+i e a_{\mu}$ is the covariant derivative acting on colorless pions. The chiral potential is

$$
V_{\phi}(\sigma, \vec{\pi})=\frac{\lambda}{4}\left(\sigma^{2}+\vec{\pi}^{2}-v^{2}\right)^{2}-h \sigma, \quad h=f_{\pi} m_{\pi}^{2}, \quad v^{2}=f_{\pi}^{2}-m_{\pi}^{2} / \lambda, \quad \lambda=20,
$$

where $f_{\pi} \approx 93 \mathrm{MeV}$ and $m_{\pi} \approx 138 \mathrm{MeV}$. The constituent quark mass is given by $m_{q} \equiv m_{q}(\langle\sigma\rangle)=$ $g\langle\sigma\rangle$, and, choosing $g=3.3$ at $T=0$, one obtains for the constituent quarks in the vacuum $m_{q} \approx$ $310 \mathrm{MeV}$. At low temperatures quarks are not excited, and the model reproduces results from the usual linear $\sigma$-model without quarks.

The Polyakov potential is given by [7]

$$
\frac{V_{L}(L, T)}{T^{4}}=-\frac{L^{*} L}{2} \sum_{l=0}^{2} a_{l}\left(\frac{T_{0}}{T}\right)^{l}+b_{3}\left(\frac{T_{0}}{T}\right)^{3} \ln \left[1-6 L^{*} L+4\left(L^{* 3}+L^{3}\right)-3\left(L^{*} L\right)^{2}\right],
$$



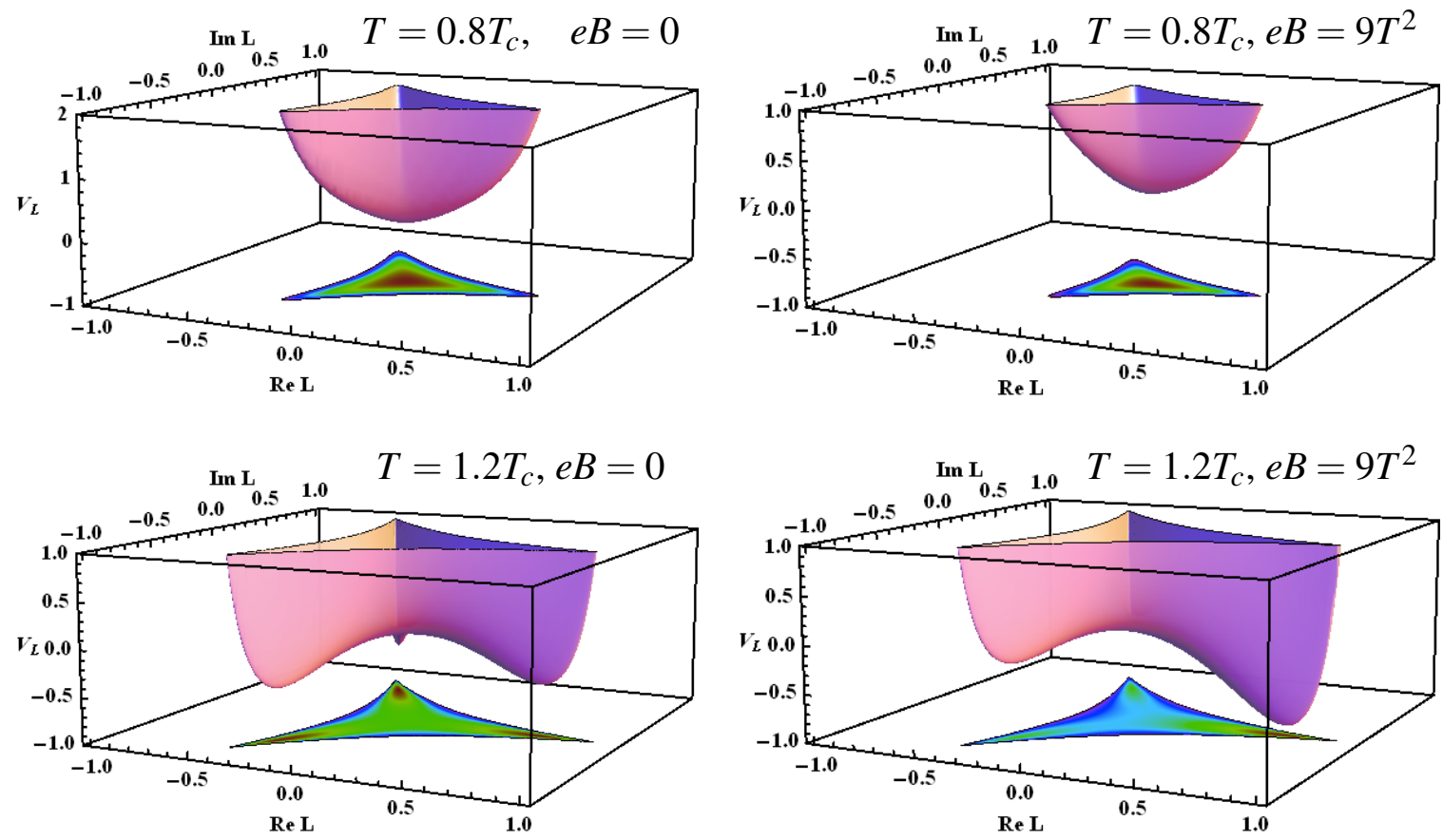

Figure 1: Effects of temperature and magnetic field on quark confinement: The Polyakov loop potential at $T=0.8 T_{0}$ (top) and $T=1.2 T_{0}$ (bottom) and at zero magnetic field (left) and at $e B=9 T^{2}$ (right).

where $T_{0} \equiv T_{S U(3)}=270 \mathrm{MeV}$ is the critical temperature in the pure gauge case and $a_{0}=16 \pi^{2} / 45 \approx$ $3.51, a_{1}=-2.47, a_{2}=15.2$, and $b_{3}=-1.75$. Below we follow a mean-field analysis in which the mesonic sector is treated classically whereas quarks represent fast degrees of freedom.

In the confining sector, the strong magnetic field affects the potential for the expectation value of the Polyakov loop via the intermediation of the quarks in three ways [5]:

(i) the presence of the magnetic field intensifies the breaking of the global $\mathbb{Z}_{3}$ symmetry and makes the Polyakov loop real-valued (see Fig. 1);

(ii) the thermal contribution from quarks tends to destroy the confinement phase by increasing the expectation value of the Polyakov loop;

(iii) on the contrary, the vacuum quark contribution tends to restore the confining phase by lowering the expectation value of the Polyakov loop.

The vacuum correction from quarks has a crucial impact on the phase structure. If one ignores the vacuum contribution from the quarks, then one finds that the confinement and chiral phase transition lines coincide, and in this case the increasing magnetic field lowers the common chiralconfinement transition temperature [5]. However, if one includes the vacuum contribution, then the confinement and chiral transition lines split, and both chiral and deconfining critical temperatures become increasing functions of the magnetic field, Fig. 2. The vacuum contribution from the quarks affect drastically the chiral sector as well. Our calculations also show that the vacuum contribution seems to soften the order of the phase transition: the first-order phase transition - which would be realized in the absence of the vacuum contribution - becomes a smooth crossover in the system with vacuum quark loops included. 

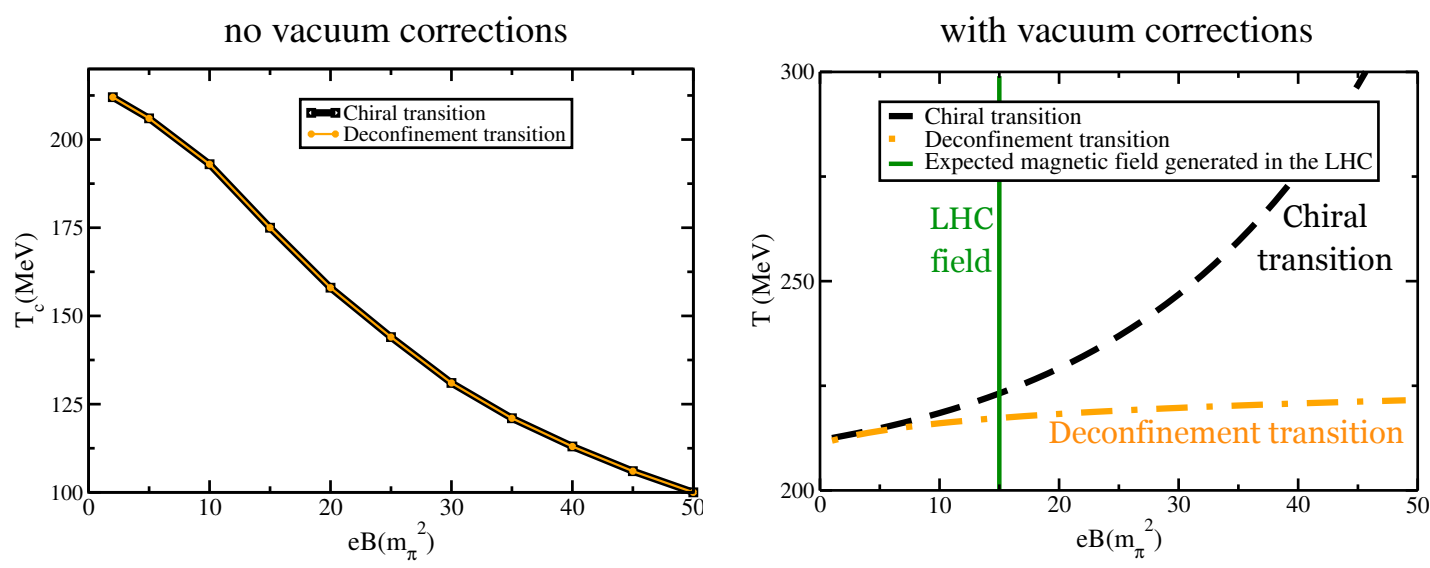

Figure 2: Phase diagram in the $B-T$ plane. (left) Without vacuum corrections: the critical temperatures of the deconfinement (the dash-dotted line) and chiral (the dashed line) transitions coincide all the way, and decrease with $B$. (right) With vacuum corrections: the critical temperatures of the deconfinement (the dashdotted line) and chiral (the dashed line) coincide at $B=0$ and split at higher values of the magnetic field. A deconfined phase with broken chiral symmetry appears. The vertical line represents a typical magnitude of the magnetic field that expected to be realized at LHC heavy-ion collisions [8].

Either scenario is very exciting, and brings new possibilities for the phase diagram of strong interactions: a first-order order transition, the splitting of coexistence lines, new phases, the explicit breaking of the center $\mathbb{Z}_{3}$ symmetry by the magnetic field, and so on.

An independent investigation in the Nambu-Jona-Lasinio model agrees with the second scenario [6], which was expected since by construction this model includes quark degrees of freedom in the vacuum. Preliminary numerical simulations of lattice QCD [9] seems to favor the second scenario as well indicating that the critical temperatures of both transitions are rising with strength of the background magnetic field.

\section{Acknowledgments}

This work was partially supported by CAPES-COFECUB, CNPq, FAPERJ, and FUJB/UFRJ.

\section{References}

[1] N. O. Agasian and S. M. Fedorov, Phys. Lett. B 663, 445 (2008).

[2] E. S. Fraga and A. J. Mizher, Phys. Rev. D 78, 025016 (2008).

[3] J. K. Boomsma and D. Boer, Phys. Rev. D 81, 074005 (2010).

[4] K. Fukushima, M. Ruggieri and R. Gatto, Phys. Rev. D 81, 114031 (2010).

[5] A. J. Mizher, M. N. Chernodub and E. S. Fraga, Phys. Rev. D 82, 105016 (2010).

[6] R. Gatto and M. Ruggieri, Phys. Rev. D 82, 054027 (2010). [arXiv:1007.0790 [hep-ph]].

[7] S. Roessner, T. Hell, C. Ratti and W. Weise, Nucl. Phys. A 814, 118 (2008).

[8] V. Skokov, A. Y. Illarionov and V. Toneev, Int. J. Mod. Phys. A 24, 5925 (2009).

[9] M. D’Elia, S. Mukherjee and F. Sanfilippo, Phys. Rev. D 82, 051501 (2010). 